\title{
Technological Opportunity, Long-Run Growth, and Convergence
}

J. Growiec and I. Schumacher

Discussion Paper 2007-34

Département des Sciences Économiques

de l'Université catholique de Louvain 
CORE DISCUSSION PAPER

2007/57

\title{
TECHNOLOGICAL OPPORTUNITY, LONG-RUN GROWTH, AND CONVERGENCE
}

\author{
Jakub GROWIEC* Ingmar SCHUMACHER ${ }^{\dagger}$
}

July 2007

\footnotetext{
*Warsaw School of Economics, Warsaw, Poland, and Center for Operations Research and Econometrics (CORE), Université catholique de Louvain, Belgium. E-mail: jakub.growiec@sgh.waw.pl.

${ }^{\dagger}$ Center for Operations Research and Econometrics (CORE), Université catholique de Louvain, Belgium. E-mail: schumacher@core.ucl.ac.be.

We are grateful to Raouf Boucekkine, Thierry Bréchet, Claude d'Aspremont, Christian Groth, Katheline Schubert, and Cees Withagen for their valuable comments, as well as to the participants of the Seminar in IMW at the University of Bielefeld. The first author acknowledges financial support from the EDNET Marie Curie fellowship program and the Foundation for Polish Science. The second author acknowledges financial support from the Chaire Lhoist Berghmans in Environmental Economics.

This text presents research results of the Belgian Program on Interuniversity Poles of Attraction initiated by the Belgian State, Prime Minister's Office, Science Policy Programming. The scientific responsibility is assumed by the authors.
} 


\begin{abstract}
We derive an R\&D-based semi-endogenous growth model where technological progress depends on the available amount of technological opportunity. Incremental innovations provide direct increases in the knowledge stock but they reduce technological opportunity and thus the potential for further improvements. Technological opportunity can be renewed only by radical innovations (which have no direct impact on factor productivity).

Investigating the model for its implications on economic growth leads to two basic observations. One, in the long-run, a balanced growth path with a constant and semi-endogenous long-run economic growth rate exists only in a specific knife-edge case which implies that technological opportunity and knowledge grow at equal rates. Two, the transition need not be monotonic. Specifically, we show under which conditions our model generates endogenous business cycles via complex dynamics without uncertainty.
\end{abstract}

Keywords and Phrases: technological opportunity, incremental innovation, radical innovation, endogenous business cycles, balanced growth, Andronov-Hopf bifurcation, complex dynamics.

JEL Classification Numbers: E32, O30, O41. 


\section{Introduction}

This article is centered around the following two questions. First, we ask how one could characterize the opportunity for new innovations, and in what ways this opportunity could evolve over time. Second, we inquiry under what circumstances the growth implications of models featuring technological opportunity correspond to those found in the endogenous (and semi-endogenous) growth literature up to now.

The mainstream literature on $\mathrm{R} \& \mathrm{D}$ and growth assumes that the amount of newly created knowledge depends primarily on the number of active researchers and on the current stock of knowledge (Romer [27], Jones [15]). The list of other potential, additional factors appearing in the knowledge accumulation function includes, among others, human capital (Strulik [32]), spillovers (Howitt [14]), R\&D expenditures and R\&D difficulty (Segerstrom [30]). It is presumed that as long as all these ingredients are supplied in required quantities, the opportunity for innovations is unlimited.

Our intention here is to work out when these standard characterizations are sufficient for understanding the sources of economic growth and drawing policy implications, and under what circumstances one ought to be more careful. We are looking for a more precise description of the opportunity for innovations, which underlies the aggregate R\&D output. In the quest for such, we shall provide one potential characterization of technological opportunity and investigate its consequences for economic growth and the transition path. Our approach bases upon the assumption that the R\&D sector produces two inherently different kinds of innovations, namely incremental and radical innovations, which are related as follows. Incremental innovations are the backbone of the economy's productivity and utilize technological opportunity opened up by radical innovations. Radical innovations extend the existing technological opportunity by combining previous discoveries (abstract ideas which are initially useless) with existing knowledge. Technological opportunity behaves like a renewable resource here: it is exhausted by incremental innovations and renewed by radical innovations. This characterization is based upon Olsson [23]. However, we extend his work in several crucial ways, the reasons for which will become clearer in the next paragraphs.

The main results of our analysis are the following. Under a rather restrictive knife-edge case, which implies that knowledge and technological opportunity grow at equal rates in the long run, our approach delivers long-run 
predictions very much along the lines of the semi-endogenous growth literature (Jones [15], Kortum [21], Segerstrom [29]). However, in addition to the standard result of monotonic convergence, we obtain the possibility of complex dynamics for a wide variety of parameter values. These complex dynamics can come in the form of converging (or diverging) oscillations or even Andronov-Hopf bifurcations. As opposed to the standard models in business cycle theory our model does not require uncertainty to generate the endogenous cycles.

We investigate the long-run implications of relaxing the specific knife-edge assumption in section 4. For example, one of the findings is that economic growth will come to a halt if the amount of radical innovations is too low in comparison to the number of incremental innovations. The amount of and changes in technological opportunity therefore are decisive for the long-run growth implications.

The main result of Olsson's [23] framework is that a linearity assumption is the source of oscillatory dynamics. This linearity assumption implies that workers either work in the incremental or the radical innovation sector, but never in both at the same time. Intuitively, this is not a convincing result: even while this incremental innovation paper is being written, radical innovations across the globe take place. Nevertheless, even though we allow for, among others, decreasing returns to research effort, which implies (optimal) research in both $R \& D$ sectors at the same time, we are still able to obtain (converging, permanent or diverging) oscillations on the optimal path. However, the returns to labor in the innovation sectors must be sufficiently large in order to create permanent oscillations. This, therefore, confirms Olsson's [23] initial intuition of the importance of labor allocations in the creation of permanent cycles.

When introducing different kinds of innovations into growth models, several researchers have already suggested the possibility of cycles to occur (Aghion and Howitt [1], Cheng and Dinopoulos [6], Amable [2], Francois and Lloyd-Ellis [8], Phillips and Wrase [25], Jovanovic and Rob [19], Bresnahan and Trajtenberg [4], Freeman, Hong and Peled [9]). However, their approaches and therefore their implied sources of cyclical growth differ from ours. In particular, in none of these approaches does technological progress depend on an exhaustible factor, as technological opportunity is in our case. We shall provide a comparison of our approach to these articles in section 3.2 .

The remainder of the article is structured as follows. In section 2 we lay 
out the foundations of our framework. In section 3 we set up and solve the basic growth model. In section 4 we discuss some generalizations of our basic model and other related issues. Section 5 concludes.

\section{Technological Opportunity and the Evolu- tion of Knowledge}

There exists a large amount of anecdotal evidence which can be used to support the view that technological opportunity is a relevant concept and that the distinction between incremental and radical innovations is vital for the proper understanding of technological change across centuries.

Example 1. In 1814, Joseph Niépce invented the first photo camera. It took 8 hours to take one picture. This certainly must be viewed as a radical innovation but it was not yet a useful technology for increasing the productivity of the economy. However, in 1851 the exposure time was reduced to 2-3 seconds, in 1888 the first roll-film was developed, and in 1941 - the color film. One and a half century later, we have digital cameras which are available at prices accessible to the general public and pictures can be printed at home. It is clear that the small improvements to the photo camera, in our terminology the incremental innovations, ought to be viewed as the crucial steps for spreading the technology into the economy, whereas the radical innovation of Joseph Niépce was the one which opened up the opportunity for these incremental innovations.

Example 2. The first locomotive was developed in 1804 by Richard Trevithick. It was the first steam-powered locomotive, and therefore ought to be considered a radical innovation. However, it was too heavy and even broke the very own rails it was supposed to travel on. Compared to this, the incremental innovations following that radical innovation were tremendous. In 1814 came the first steam locomotive that was actually able to travel, although at only $6 \mathrm{~km} / \mathrm{h}$; today the Maglev, the high-speed magnetic train, travels at more than $550 \mathrm{~km} / \mathrm{h}$. Again, the initial idea of Richard Trevithick was the one opening up the possibilities for the incremental innovations, whereas the radical innovation proved useless for improving productivity.

Example 3. In 1928, Alexander Fleming had, by accident, left a Staphylococcus plate culture lying in the warm cellar. Several days later, upon reminding himself of the forgotten plate culture, he noticed that there was a 
blue-green colored mold destroying the bacteria. He called this mold Penicillin. It was however too weak and unstable to provide a useful means of destroying bacterial infections in humans. Only subsequent research by Chain, Florey and Heatley developed the kind of Penicillin which now saves human lives throughout the world. As Sir Henry Harris had aptly put it: "Without Fleming, no Chain; without Chain, no Florey; without Florey, no Heatley; without Heatley, no Penicillin."

Similar stories can be told about the invention of the first battery by Alessandro Volta in 1799, the first champagne by Dom Pérignon in 1670, nylon by DuPont in 1928, the steam engine, the airplane, electricity, and many more. What can be seen here is that we view radical innovations as spanning a broader class of innovations than for example General Purpose Technologies (e.g. Jovanovic and Rousseau [20]). However, they all have something in common: they all open up opportunities for small improvements, for incremental innovations that help make the technology accessible, practical and operative. Without the radical innovations being able to open up new opportunities, there would be no place for incremental innovations. And without doubt, in a considerable if not exhaustive number of cases, it were the incremental innovations which really proved to be useful for economic purposes.

Our understanding of the evolution of technological knowledge differs also slightly from the articles basing on combinatorial calculations (Romer [28], Weitzman [35]). We do not consider the potential for technological change as the number of possible ways to combine ideas. For example, 20 objects may be combined in $2^{20}=1,048,576$ ways. Given an enormous number like this, papers in this vein conclude that there are no practical limits to technological change. However, why should we combine every possible idea? Intuitively, it seems more likely that mostly the latest technologies, those on the technological frontier (Caselli and Coleman [5]), are improved. The importance of the technological frontier can be supported e.g. by the Poincaré's observation: "To create consists precisely in not making useless combinations." So, we suggest that only ideas on the technology frontier may be usefully improved, an assumption which this article shares with Kortum [21], Jones [18] and Olsson [23]. The implication is that incremental innovations would by themselves come to a halt if the technological frontier were not constantly pushed ahead by radical innovations.

So, how does technological opportunity increase? What qualifies as a radical innovation? We suggest that a radical innovation arises whenever a 
new class of known phenomena (physical, biological, chemical, etc.) is found to have economically useful applications. For example, "[f] or thousands of years, silicon dioxide provided utility mainly as sand on the beach, but now it delivers utility through the myriad of goods that depend on computer chips" (Jones [17]). Conclusively, we believe that the flows of radical innovations are related to the stock of existing knowledge. The larger the existing knowledge base, the more discoveries will be transformed into radical innovations. Now being equipped with the basic understanding of how we understand the evolution of technology, we will commence to model the relationships above.

\subsection{The Laws Of Motion}

Let us now provide some notation. By $B(t)$ we shall denote the amount of technological opportunity at time $t$, by $R(t)$ - the flow of radical innovations, by $C(t)$ - the flow of incremental innovations, and by $A(t)$ - the current stock of knowledge. Following the preceding arguments we assume that technological opportunity is increased by radical innovations, whereas incremental innovations add to the stock of knowledge but diminish technological opportunity. We thus write $\dot{A}(t)=C(t)$ and $\dot{B}(t)=R(t)-C(t)$.

We are now equipped with the basic understanding of how innovations and knowledge evolve over time, and we shall proceed to provide a more specific characterization which would then allow us to solve for the precise dynamic paths. To assure analytical tractability as well as comparability to the semi-endogenous growth literature which evolved from Jones [15], we use standard Cobb-Douglas functional forms. Omitting time arguments for convenience, these read:

$$
\begin{aligned}
& \dot{A}=\delta\left(u \ell_{A} L\right)^{\beta} B^{\mu}, \\
& \dot{B}=\underbrace{-\delta\left(u \ell_{A} L\right)^{\beta} B^{\mu}}_{\text {incremental innovations }}+\underbrace{\gamma\left((1-u) \ell_{A} L\right)^{\beta} A^{\nu}}_{\text {radical innovations }},
\end{aligned}
$$

We denote total population by $L$ and assume it to be equal to the total amount of working time available in the economy at time $t$. Population is assumed to grow exogenously at constant rate $n>0$. Then, $\ell_{A}$ is the proportion of working time devoted to $\mathrm{R} \& \mathrm{D}$, with $u \ell_{A}$ being the proportion of time spent on working in the incremental innovation sector and $(1-u) \ell_{A}$ the time spent in the radical innovation sector. The parameter $\delta>0$ is proportional to the rate at which incremental innovations come about, whereas $\gamma>0$ 
relates to the rate at which radical innovations arrive. The exponents $\mu$ and $\nu$ are crucial for the dynamic behaviour of our model. From our preceding argumentation, we know that they ought to be strictly positive. We shall further assume that $\mu, \nu \in(0,1)$ which assures non-explosive semi-endogenous growth in the long run. The relative size of these exponents is decisive for the long-run evolution of innovations, which we investigate in the next subsection. The parameter $\beta>0$ measures the degree of returns to scale in the R\&D sectors. Our assumption that $\beta \in(0,1)$ implies decreasing returns to scale in R\&D activity which are required for positive shares of both kinds of innovation to be pursued in optimum and stands in contrast to Olsson [23] who has $\beta=1$ and thus constant returns to scale, which leads to bang-bang solutions.

We contrast the setup of our model with several standard R\&D-based models of (semi-)endogenous growth. Romer's [27] specification of technical change would correspond to equation (1) only, with $B=A$ and $\mu=1$. This of course leads to the scale effect discussed by Jones [15] and implies explosive dynamics if $n>0$. However, even the Jones's R\&D equation, which has $\mu<1$, is only a subcase of our specification. In comparison to Olsson [23], we allow for both incremental and radical innovations happening at the same time. We shall explain what is the direct implication of considering the additional feedback loop through technological opportunity. Generally, it gives two new results. Firstly, the long-run results of e.g. Jones [15] are preserved only when the specific knife-edge condition $\mu=\nu$ holds, and secondly, the optimal path can be subject to oscillations. Our model is thus able to span a bridge from the oscillatory transition of Olsson to the monotonic dynamics of Jones or Romer. ${ }^{1}$ Let us analyze the long-run implications first.

\subsection{Long-Run Evolution}

We shall now focus on the properties of the balanced growth path (BGP) under different relative sizes of the exponents in the radical and incremental innovations, $\mu$ and $\nu$ (or of the asymptotic balanced growth path, if a proper BGP does not exist). ${ }^{2}$ We shall do this by finding the necessary conditions

\footnotetext{
${ }^{1}$ Dynamics of the Jones' [15] model have been analyzed in detail by Arnold [3]. Perhaps a bit surprisingly, he finds that the possibility of oscillatory convergence to the BGP cannot be ruled out there.

${ }^{2}$ When we refer to semi-endogenous growth we mean growth ultimately driven by population growth, see e.g. Jones [15].
} 
under which the growth rates of all economic variables are constant. These conditions imply in particular that the sectoral allocation of labor does not change over time.

Let us take equations (1)-(2) and solve for the BGP. It is easy to see that the growth rate of technological opportunity, $\dot{B} / B=R / B-C / B$ is constant only if both the radical and incremental innovation flows grow at the same rate as technological opportunity itself does. This is the case if $\mu=\nu$. When this condition is satisfied, it implies that both knowledge and technological opportunity grow at the same rate, too. As can be observed from equation (2), the growth rates of $C$ and $R$ are different if the exponents $\mu$ and $\nu$ are not equal. The implications are summarized in the following table.

\begin{tabular}{|c|c|c|}
\hline \multicolumn{3}{|c|}{ Long-Run Behavior of $A$ and $B$} \\
\hline Case 1 & $\mu=\nu$ & $\hat{A}=\hat{B}=\frac{\beta n}{1-\mu}$ \\
\hline Case 2 & $\mu<\nu$ & $\lim _{t \rightarrow \infty} \hat{B}=\frac{1+\nu}{1+\mu} \hat{A}$ \\
\hline Case 3 & $\mu>\nu$ & $\lim _{t \rightarrow \infty} \dot{A}=\dot{B}=B=0$ \\
\hline
\end{tabular}

The intuition is as follows. In Case 1 we observe that if our knife-edge condition $\mu=\nu$ is satisfied, and in consequence, the extents of external returns from technology to radical innovations and from technological opportunity to incremental innovations are equal, then this gives rise to standard semi-endogenous growth along the lines of Jones [15]. Unfortunately, this knife-edge condition is crucial for the result. This can be seen from Case 2 and Case 3. In Case 2, the external returns to radical innovations are larger than to incremental ones, and thus technological opportunity can grow at a faster rate than knowledge. Clearly, in the limit, technological opportunity will be exhaustively driven by radical innovations in that case. This is still not so bad news for the economy in comparison to Case 3. In such case, even though technological opportunity is renewed by radical innovations, the effect of its depletion due to more efficient incremental innovations is dominant in the long run. Growth comes to a halt in the limit.

Let us remark here that if one allows for different degrees of decreasing returns to scale in the two $\mathrm{R} \& \mathrm{D}$ sectors, such that $\dot{B}=-\delta\left(u \ell_{A} L\right)^{\beta} B^{\mu}+$ $\gamma\left((1-u) \ell_{A} L\right)^{\xi} A^{\nu}$, the crucial knife-edge condition $\mu=\nu$ translates into a different knife-edge condition: $\beta /(1-\mu)=\xi /(1-\nu)$. This condition has exactly the same interpretation: flows of incremental and radical innovations are supposed to grow at equal rates. Further on, the counterpart for Case 
2 is $\beta /(1-\mu)<\xi /(1-\nu)$, and the counterpart for Case 3 is $\beta /(1-\mu)>$ $\xi /(1-\nu)$. All further implications are preserved. We shall abstract from this unnecessary complexity throughout the remainder of the article.

It is now clear that if technological opportunity is accepted to be the driving force behind the possibility to innovate, then the long-run behavior of the economy depends crucially on the relative size of external returns to different kinds of innovations. In consequence, long-run predictions along the lines of Jones [15], Kortum [21], Segerstrom [29] or Peretto [24] hold only in the case where the extents of external returns to incremental and radical innovations are exactly equal. Since current literature compares the model predictions with historical time paths of $\mathrm{R} \& \mathrm{D}$ expenditures and factor productivities, with the single dynamical equation of technology in mind, our knife-edge result here suggests one should be more cautious with those predictions. Given one accepts technological opportunity as the driving force behind actual technology, then it could very well be that the empirical literature misses the influence of the evolution of technological opportunity on effective technological progress and thus runs into systematic error.

As our main interest lies in elaborating the implications of introducing technological opportunity into a standard semi-endogenous growth framework, we shall concentrate mostly on the case $\mu=\nu$ which allows for a BGP. As we will show, even in the knife-edge case we will obtain novel results, this time for the transition.

\section{The Social Planner Problem}

Let us now embed the dynamical equations analyzed above in a semi-endogenous growth model and find its social planner solution. Our interest is to understand in which way will the planner allocate consumption across time and labor across sectors in order to achieve the social optimum. We shall base on the standard Ramsey framework, where the infinitely-lived representative agent obtains utility from the discounted stream of a single consumption good. The utility function takes the CRRA form: $u(c)=c^{1-\theta} /(1-\theta)$, with $\theta>0$ being the inverse of the intertemporal elasticity of substitution in consumption. Moreover, we shall assume a standard Cobb-Douglas production function which takes as inputs: technology $A$ with elasticity $\sigma$, physical capital $K$ with elasticity $\alpha$, and labor $\left(1-\ell_{A}\right) L$ with elasticity $1-\alpha$. Technology accumulates faster the more labor is allocated to research, but its underlying 
possibility to accumulate is constrained by technological opportunity.

As suggested previously, we shall concentrate on the case of $\mu=\nu$ here. We impose this restrictive condition for analytical tractability and comparability to the semi-endogenous growth literature. We shall first solve for the BGP of the system. The maximization problem looks as follows.

$$
\begin{aligned}
\max _{\left\{c, u, \ell_{A} ; k, A, B\right\}_{t=0}^{\infty}} & L_{0} \int_{0}^{\infty} \frac{c^{1-\theta}}{1-\theta} e^{-(\rho-n) t} d t \quad \text { subject to: } \\
y= & A^{\sigma} k^{\alpha}\left(1-\ell_{A}\right)^{1-\alpha} \\
\dot{k}= & y-c-(d+n) k, \\
\dot{A}= & \delta\left(u \ell_{A} L\right)^{\beta} B^{\mu} \\
\dot{B}= & {\left[-\delta u^{\beta} B^{\mu}+\gamma(1-u)^{\beta} A^{\mu}\right]\left(\ell_{A} L\right)^{\beta}, } \\
L= & L_{0} e^{n t}, \quad n>0, \\
& L_{0}, k_{0}, A_{0}, B_{0} \text { given. }
\end{aligned}
$$

The parameter restrictions are $0<n<\rho$, necessary to guarantee a positive effective discount rate, and $\sigma, \alpha, \beta, d \in(0,1)$ as well as $\theta, \mu, \delta, \gamma>0$. Finally, $\mu<1$ is required to guarantee positive semi-endogenous growth in the longrun. $d$ is the instantaneous depreciation rate of physical capital.

\subsection{The Balanced Growth Path}

Maximizing the Hamiltonian associated with the above optimization problem and solving for the BGP yields the following long-run growth rate of the economy:

$$
g \equiv \hat{y}=\hat{k}=\hat{c}=\frac{\sigma}{1-\alpha} \frac{\beta n}{1-\mu}
$$

whereas the long-run growth rates of technology and technological opportunity are (as in Jones [15]):

$$
\hat{A}=\hat{B}=\frac{\beta n}{1-\mu} .
$$

As expected from the previous section, technological opportunity and technology grow at a common rate, and consumption, income and capital grow 
at a rate being a multiple of this rate. Along the BGP, shadow prices our state variables evolve according to:

$$
\begin{aligned}
\hat{\lambda}_{k} & =n-\rho-\theta g \\
\hat{\lambda}_{A}=\hat{\lambda}_{B} & =n-\rho-\left(\theta-1+\frac{1-\alpha}{\sigma}\right) g,
\end{aligned}
$$

and thus all transversality conditions boil down to the single requirement that $n<\rho+(\theta-1) g$.

Denoting the steady-state ratio of technology to technological opportunity $A / B$ by $X$, we find that the optimal share of incremental research effort relative to radical research effort is:

$$
u^{*}=\frac{1}{1+F^{\frac{1}{1-\beta}} X^{\frac{1+\mu}{1-\beta}}\left(\frac{\gamma}{\delta}\right)^{\frac{1}{1-\beta}}},
$$

where $X \equiv A / B$ solves the following implicit equation:

$$
\left(\frac{\gamma}{\delta}\right)^{\frac{1}{1-\beta}} F^{\frac{\beta}{1-\beta}}(X)^{\frac{1+\mu}{1-\beta}}=1+X
$$

with $F=-\frac{\mu \hat{A}}{\hat{\lambda}_{A}}>0$. It is straightforward to show that (14) always has a unique positive solution. The argument goes as follows. The right-hand side of equation (14) as a function of the ratio $X$ increases linearly from $\lim _{X \rightarrow 0}=1$ to $\lim _{X \rightarrow \infty}=\infty$. The left-hand side increases in a strictly convex manner from $\lim _{X \rightarrow 0}=0$ to $\lim _{X \rightarrow \infty}=\infty$. Since both sides are continuous, there necessarily exists a unique, positive point in which they intersect.

Furthermore, we find that the optimal share of labor allocated to R\&D along the BGP is

$$
\ell_{A}^{*}=\frac{1}{1+u^{*} \frac{(1-\alpha) \mu}{\beta \sigma}\left(\Phi X-\frac{1-u^{*}}{u^{*}}\right)}
$$

where

$$
\Phi=1+\frac{\gamma}{\delta}\left(\frac{1-u^{*}}{u^{*}}\right)^{\beta-1} X^{\mu} .
$$

The interpretation of these results is as follows. The determinants of the optimal sectoral allocation of labor are, among others: 
(i) the growth rate of technology relative to the growth rate of its shadow price. The higher the growth rate of technology, the more worthwhile it is to increase technological opportunity by radical innovations;

(ii) the size of technology relative to the size of technological opportunity. The larger is technological opportunity, the more researchers should be allocated to incremental innovations and the more workers should be allocated to the overall $\mathrm{R} \& \mathrm{D}$ sector;

(iii) the ratio of the exogenous intensities of producing incremental and radical innovations, captured by $\gamma$ and $\delta$ respectively. The higher is the chance of coming up with a radical innovation, the more researchers should be allocated to radical innovations, and vice versa;

(iv) the elasticity parameters. The smaller is the technology share in production (smaller $\sigma$ ), the less efficient researchers are (smaller $\beta$ ), or the smaller is the extent of external effects in $\mathrm{R} \& \mathrm{D}$ (smaller $\mu$ ), the less workers will be employed in research;

(v) the effect of radical innovations creating an additional demand for research. The more researchers are allocated in the radical innovation sector (the smaller $u^{*}$ ), the more researchers are employed in overall $\mathrm{R} \& \mathrm{D}$ (larger $\left.\ell_{A}^{*}\right)$.

\subsection{The Transition}

In this section we shall analyze the transition dynamics of our model around the balanced growth path. We derive the dynamical equations for variables which are stationary along the BGP. Hence, our system is going to be rewritten in terms of the six following variables: $\left\{c / k, u, \ell_{A}, y / k, A / B, L^{\beta} / A^{1-\mu}\right\}$. We shall again denote $X \equiv A / B$, and use also the notation $Y \equiv L^{\beta} / A^{1-\mu}$.

The transition dynamics are fully characterized by the following dynam- 
ical system.

$$
\begin{aligned}
\widehat{c / k}= & \left(\frac{\alpha}{\theta}-1\right) \frac{y}{k}-\frac{d+\rho}{\theta}+\frac{c}{k}+d+n \\
\widehat{y / k}= & \sigma \delta\left(u \ell_{A}\right)^{\beta} X^{-\mu} Y+(\alpha-1)\left(\frac{y}{k}-\frac{c}{k}-d-n\right)-(1-\alpha)\left(\frac{\ell_{A}}{1-\ell_{A}}\right) \hat{\ell}_{A} \\
\hat{X}= & \delta\left(u \ell_{A}\right)^{\beta} X^{-\mu} Y+\delta\left(u \ell_{A}\right)^{\beta} X^{1-\mu} Y-\gamma\left((1-u) \ell_{A}\right)^{\beta} X Y \\
\hat{Y}= & \beta n-(1-\mu) \delta\left(u \ell_{A}\right)^{\beta} X^{-\mu} Y \\
\hat{u}= & -\frac{(1-u)}{1-\beta}\left\{\mu \hat{X}+\left[\frac{\beta \sigma}{1-\alpha}\left(\frac{1-\ell_{A}}{u \ell_{A}}\right)-\mu X\left(1+\frac{\gamma}{\delta}\left(\frac{1-u}{u}\right)^{\beta-1} X^{\mu}\right)+\right.\right. \\
& \left.\left.+\mu\left(\frac{1-u}{u}\right)\right] \delta\left(u \ell_{A}\right)^{\beta} X^{-\mu} Y\right\} \equiv-\frac{(1-u)}{1-\beta} \cdot \Xi \\
\hat{\ell}_{A}= & \frac{1}{1-\beta+\alpha \frac{\ell_{A}}{1-\ell_{A}}}\left\{\alpha \frac{c}{k}-(1-\alpha)(d+n)+\beta n+(\mu-\sigma) \delta\left(u \ell_{A}\right)^{\beta} X^{-\mu} Y+\right. \\
& \left.-\mu\left(\frac{u}{1-u}\right) \gamma\left((1-u) \ell_{A}\right)^{\beta} X Y-u \cdot \Xi\right\} .
\end{aligned}
$$

In the remainder of this section, we shall resort to numerical approximations because the implied analytical formulas, although readily attainable, are too large to be informative.

We assign baseline values to all parameters in our model. To bring our numerical example as close to reality as possible, we shall pick these values within a calibration exercise. This we do by drawing on a similar calibration exercise carried out by Steger [31] for a generalized R\&D-based semiendogenous growth model. Following him, we choose $\sigma=0.3, \alpha=0.36, d=$ $0.04, \rho=0.05, n=0.015, \mu=0.5, \beta=0.512$, parameters which have also been used in Eicher and Turnovsky [7] and other articles. We also pick $\theta=2$ which roughly corresponds to the empirical estimates of the intertemporal elasticity of substitution in consumption for developed economies, even though the debate around this value has not yet been settled.

Further parameters, $\gamma$ and $\delta$, have clearly never had the opportunity to be discussed in the literature. Thus, we pick them at arbitrary plausible values and then discuss what happens if we manipulate them. All the calibrated parameters are listed in Table 1.

For the baseline calibration of our model, we obtain the following set of BGP characteristics (Table 2). Please note that below the actual steady- 


\begin{tabular}{|c|c|c|c|c|c|c|c|c|c|}
\hline$\delta$ & $\gamma$ & $\beta$ & $n$ & $\theta$ & $d$ & $\alpha$ & $\sigma$ & $\rho$ & $\mu$ \\
\hline 2 & 2 & .512 & .015 & 2 & .04 & .36 & .3 & .05 & .5 \\
\hline
\end{tabular}

Table 1: The baseline calibration.

state values, we included comparative statics. These are helpful for the understanding of the workings of the analyzed model. A "+" means that an increase in a given parameter raises the given steady-state value, whereas a "-" means it lowers it. 0 denotes no impact. Please note that some of these comparative statics need not hold for all parameter values; what we assure is that they must hold in the vicinity of our baseline calibration.

\begin{tabular}{|c|c|c|c|c|c|c|c|c|c|c|}
\hline & $g$ & $\hat{A}$ & $\frac{y}{k}$ & $\frac{c}{k}$ & $s$ & $\frac{A}{B}$ & $\frac{L^{\beta}}{A^{1-\mu}}$ & $u$ & $\ell_{A}$ & $\ell_{Y}$ \\
\hline & .0072 & .0154 & .2900 & .2278 & .2145 & 3.163 & .0674 & .6429 & .0688 & .9312 \\
\hline \hline$\beta$ & + & + & + & + & - & - & + & - & + & - \\
$\mu$ & + & + & + & + & - & - & + & - & + & - \\
$\alpha$ & + & 0 & - & - & + & + & - & + & + & - \\
$\sigma$ & + & 0 & + & + & - & + & - & + & + & - \\
$n$ & + & + & + & + & + & - & + & - & + & - \\
$\theta$ & 0 & 0 & + & + & - & + & - & + & - & + \\
$\gamma$ & 0 & 0 & 0 & 0 & 0 & - & - & + & + & - \\
$\delta$ & 0 & 0 & 0 & 0 & 0 & + & + & - & - & + \\
$\rho$ & 0 & 0 & + & + & - & + & + & + & - & + \\
\hline
\end{tabular}

Table 2: The social planner allocation and comparative statics.

Our intention now is to emphasize some of the particularly noteworthy facts.

(i) Technological opportunity plays a similar role to savings. In particular, increases in the discount rate $\rho$ and reductions in the intertemporal elasticity of substitution in consumption $1 / \theta$ reduce both the savings rate and technological opportunity relative to technology.

(ii) The $\beta$ parameter, measuring returns to scale in $\mathrm{R} \& \mathrm{D}$, influences the steady-state variables in the same way as the $\mu$ parameter, measuring the extent of external effects in R\&D.

(iii) Increases in the population growth rate raise long-run growth rates, but reduce the level of de-trended technology. The ratio of technology to 
technological opportunity is decreased as well. Relatively more technological opportunity goes then together with an increase in overall R\&D labor share.

(iv) Apart from the cases of parameters $\gamma$ and $\delta$ which are directly related to the arrival rates of incremental and radical innovations, technological opportunity and radical research intensity $1-u$ move together. Apart from the case of a changing discount rate $\rho$, they move in the opposite direction than the de-trended stock of knowledge.

(v) Increased dependence on physical capital (higher $\alpha$ ) triggers both a higher steady-state R\&D intensity, and a higher intensity of incremental research as compared to radical research. This lowers technological opportunity but, on balance, increases the de-trended stock of knowledge.

The most important reason why we are so explicit about the calibrated parameter values is nevertheless that we would like to show that even under these very standard parameter choices, our second main result - namely various kinds of transition along the optimal path - is easily obtained. This can be seen in the subsequent table which presents the eigenvalues of the dynamical system after its linearization around the steady state. The two complex eigenvalues with negative real parts imply that under our benchmark calibration, dampened oscillations are observed.

Furthermore, we confirm by the means of a sensitivity analysis that such oscillations indeed occur under a large variety of parameter choices. In contrast, occurrence of oscillatory dynamics is impossible in setups with semiendogenous growth but without technological opportunity, such as the one of Jones [15].

\begin{tabular}{|rl|}
\hline 0.1233 & $+0.0176 i$ \\
0.1233 & $-0.0176 i$ \\
0.0557 & \\
-0.0811 & $+0.0176 i$ \\
-0.0811 & $-0.0176 i$ \\
-0.0135 & \\
\hline
\end{tabular}

Table 3: Eigenvalues of the linearized system. 
Conclusively, if one adopts the technological opportunity approach presented here, then his model can easily generate business-cycle dynamics along the transition to the BGP. The source of these cycles is found in the relative rates at which radical and incremental innovations arrive.

The intuition for the oscillatory dynamics is as follows. Incremental innovations at the same time reduce technological opportunity and improve actual technology. This technology then feeds back into radical innovations which increase technological opportunity. If radical innovations come at amounts lower than that of incremental innovations, then the increases in technological opportunity are small, and smaller than the reductions through incremental innovations. This leads to a monotonic convergence of the optimal ratio $A / B$ to the steady-state value from above. On the contrary, if radical innovations come at relatively large amounts, then the same mechanism leads to convergence of $A / B$ from below. In the case radical innovations arrive at some intermediate number, then the improvements in technological opportunity will be counterbalanced by the reductions through incremental innovations at an amount which makes the ratio of actual technology to technological opportunity fluctuate and converge to the steady-state value of $A / B$ in a non-monotonic manner.

We can therefore conclude that growth in our model is subject to fluctuations around a trend which is ultimately driven by population growth.

We wish to provide further analysis of the complex dynamics by doing some comparative statics with respect to the decisive parameters. As is visible in the subsequent Figure 1, complex eigenvalues and thus oscillations occur for an intermediate range of arrival rates of the radical innovations relative to incremental innovations (ceteris paribus). Moreover, the oscillation frequency and thus the length of the cycles depends on the $\gamma / \delta$ ratio, with a maximum frequency appearing around $\gamma / \delta=1$ (in the Figure, this corresponds to $\gamma=\delta=2$ ). Even though not decisive for the growth rate, these two arrival rates are decisive for the transition.

To take the analysis of the model's dynamics a step further, we shall now discuss the consequences of varying the returns-to-scale parameter $\beta$ in its range $(0,1]$, as presented in Figure $2 .{ }^{3}$ In such case, not only dampened oscillations appear but also limit cycles. We identify an Andronov-Hopf bifurcation.

As can be seen in Figure 2, as long as $\beta<0.96569$, greater returns to scale

\footnotetext{
${ }^{3}$ All other parameters are set at their benchmark values.
} 


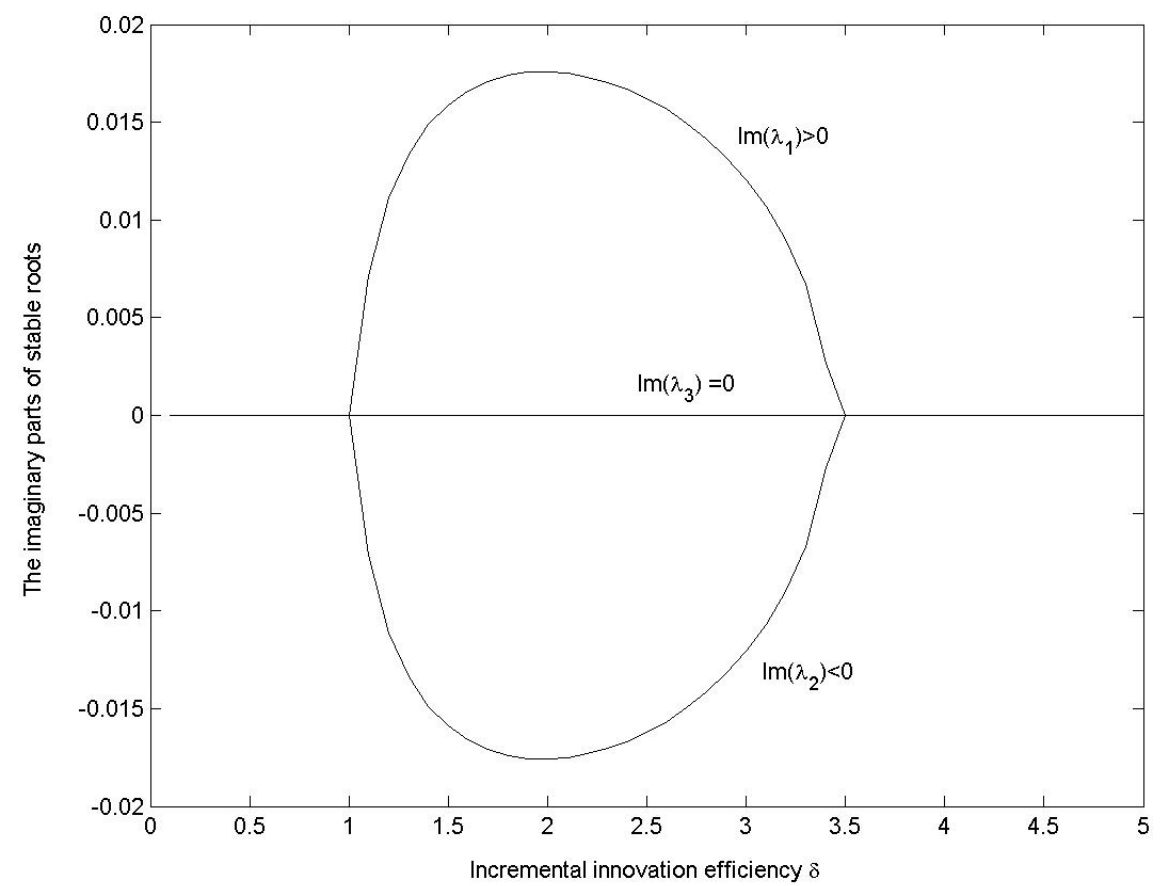

Figure 1: Oscillatory dynamics. The effect of varying $\delta$, holding $\gamma=2$ fixed.

in R\&D imply faster convergence to the BGP but also oscillations of higher frequency. When $\beta$ crosses the threshold value of 0.96569 from below, the relevant conjugate eigenvalues have their real parts crossing zero from below and there emerges a limit cycle. This means that we observe an AndronovHopf bifurcation. The intuition for this result is the following. If returns to scale in the $\mathrm{R} \& \mathrm{D}$ sector are reduced fast (low $\beta$ ), convergence to the BGP is monotonic, because $\mathrm{R} \& \mathrm{D}$ output is quickly becoming less and less responsive to labor reallocations. This curbs the incentives to constantly reallocate researchers across the two R\&D sectors and thus eliminates fluctuations. If $\beta$ is larger then dampened oscillations appear, and their frequency increases with $\beta$. If returns to scale in $R \& D$ are high or close to constant, then incentives to reallocate labor are very strong, and business cycles become persistent. Obviously, as we noticed above, the intuition for cycles does not only depend on the returns to scale to labor in the $\mathrm{R} \& \mathrm{D}$ sector, but indeed requires the two kinds of innovations to come in approximately similar amounts, too. 

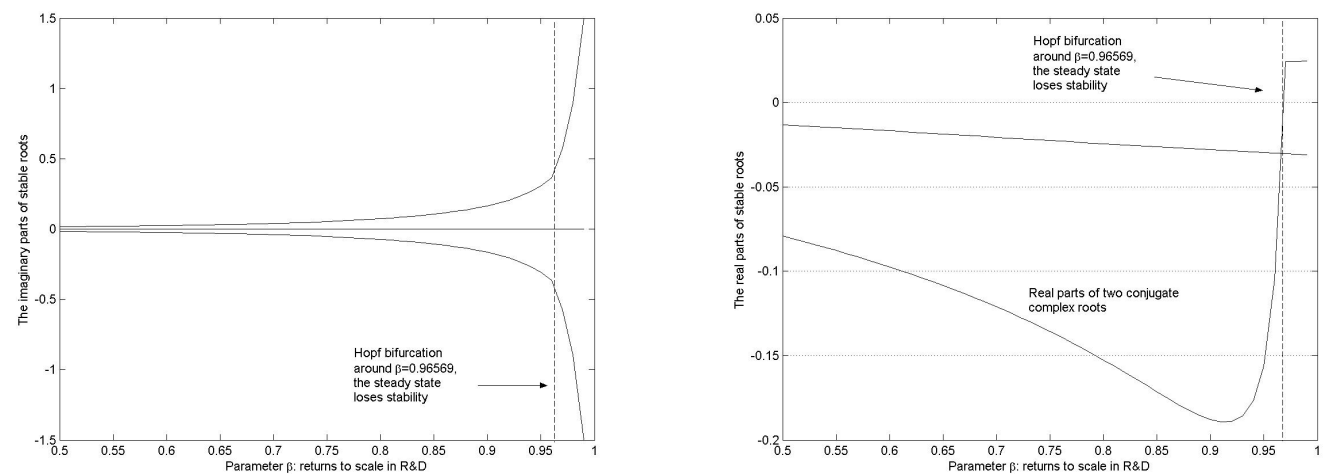

Figure 2: Changes in dynamics following changes in $\beta$. The Andronov-Hopf bifurcation appears around $\beta \approx 0.96569$.

We also notice that the Olsson's [23] initial intuition for obtaining persistent cycles, namely the linearity assumption which he imposes (here it corresponds to $\beta=1$ ), was correct. However, as we demonstrate, $\beta=1$ is not a necessary condition for cycles in our setup. Indeed, optimal permanent cycles occur already for less-than-constant returns to scale in $\mathrm{R} \& \mathrm{D}, \beta<1$, although given our baseline parameters, $\beta$ needs to be close to $1 .{ }^{4}$ Obviously, the higher is population growth, the lower the $\beta$ which leads to permanent cycles.

As suggested, even though oscillations may occur for rather low values of $\beta$, it seems that a necessary condition for limit cycles is a high value of $\beta$. That this is not sufficient will be shown now. We assign $\beta$ a value of 0.97 , close to the bifurcation value discussed above, and check the consequences of varying the R\&D spillover parameter $\mu$ as in Figure 3. The larger the spillover parameter $\mu$ the slower are the oscillations, and for large values (here $\mu>0.85)$ oscillations disappear completely. The Andronov-Hopf bifurcation occurs, of course, again and is identified at $\mu \approx 0.56$. A smaller $\mu$ suggests a relatively higher importance of labor for the creation of innovations, which implies that the preferences of the social planner have a larger influence over the path of innovations (which we confirm below). On the contrary, the larger is $\mu$ the more important become the relative amounts of technology and technological opportunity.

\footnotetext{
${ }^{4}$ This finding, of course, does not exclude the possibility that other parameter values lead to bifurcations for values of $\beta$ significantly below 1 .
} 

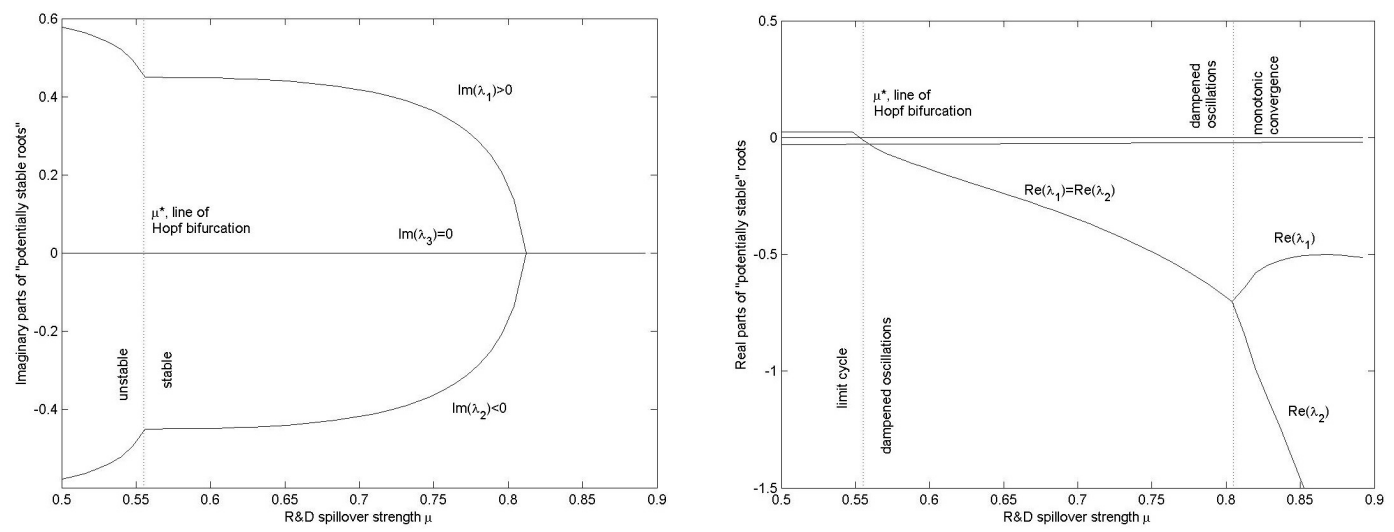

Figure 3: Changes in dynamics following changes in $\mu$. When $\beta=0.97$, the Andronov-Hopf bifurcation appears around $\mu \approx 0.56$.

We have also performed a similar analysis for changes in the discount rate, as in Figure 4. At $\rho \approx 0.053$ we observe a similar Andronov-Hopf bifurcation. When $\rho<0.053$, we have converging oscillations, and smaller values of $\rho$ lead to oscillations of smaller frequency, as intuition would suggest. This result suggests that not only technology matters, but the preferences of the planner play a role, too. So, if the planner is sufficiently impatient (large $\rho$ ) then he will initially allocate more labor to incremental innovations, allowing faster growth now and therefore more consumption. However, because radical innovations will then come in smaller amounts, and technological opportunity will be gradually exhausted, at some point the planner will have to increase the number of researchers in the radical innovations sector in order to prevent economic stagnation. In the moment that enough technological opportunity will have been created, the planner will shift the workers again to the incremental innovations sector to satisfy his impatience for consumption. In case the discount rate is too large, the economy will never converge to the BGP because the planner will be too quick in reallocating labor across the two R\&D sectors.

\subsection{Cycles: A Comparison to the Literature}

Our result of oscillatory dynamics ought to be compared to the predictions found in previous literature. The following list of contributions is obviously not exhaustive; it only serves to illustrate the differences between our ap- 

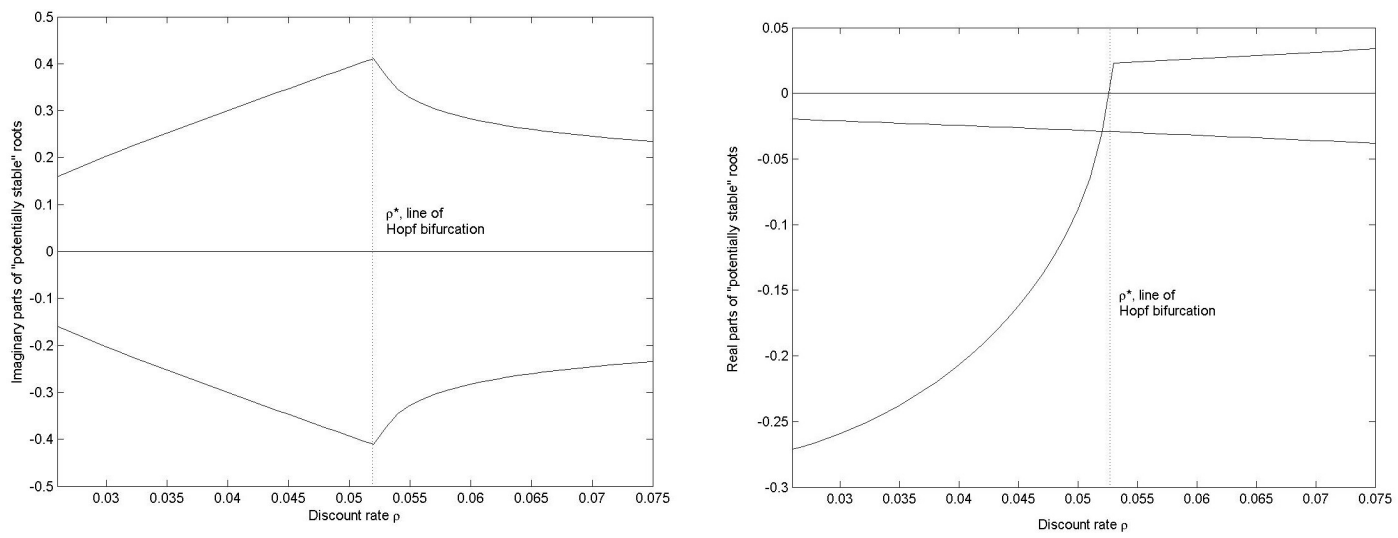

Figure 4: Changes in dynamics following changes in the discount rate $\rho$. When $\beta=0.97$, the Andronov-Hopf bifurcation appears around $\rho \approx 0.053$.

proach and the other ones. We have also grouped together articles where cycles are generated through channels which are broadly the same. In these articles, the main sources of oscillatory dynamics are:

(i) the relationship between wage costs, population growth, and profits (Goodwin [10]; Francois and Lloyd-Ellis [8]). If growth is high, then unemployment falls, raising wages and decreasing profits. When growth is lower than population growth, this leads to a recession, increases unemployment and starts the cycle again;

(ii) creative destruction (Aghion and Howitt [1]; Cheng and Dinopoulos [6]; Amable [2]; Francois and Lloyd-Ellis [8]; Phillips and Wrase [25]);

(iii) uncertainty (Kydland and Prescott [22]). This is the basic assumption of the real business cycle theory which utilizes productivity shocks and adjustment lags to generate aggregate fluctuations;

(iv) monopoly profits accrued from the distinction between fundamental and secondary innovations (Jovanovic and Rob [19]; Cheng and Dinopoulos [6]). These models are based on a similar distinction as our model is. Effectively though, the fluctuations in monopoly profits are the actual source of cycles;

(v) General Purpose Technologies - GPTs (Bresnahan and Trajtenberg [4]; Helpman and Trajtenberg [13], Freeman, Hong and Peled [9]). GPTs 
are assumed to have the property that before they can be applied, costly adaptation to them is required. This reduces economic growth in the short run but increases it afterwards;

(vi) linearity (Olsson [23]). Due to the Olsson's linearity assumption, labor is either fully allocated to radical or to incremental research. The interplay between these two types of innovations leads to cycles just like in our model, but it does so through the bang-bang labor allocation and discrete jumps in technological opportunity.

Unlike all these works, our model generates smooth endogenous cycles through the interplay between currently available knowledge and technological opportunity. Because of the sources as well as the nature of cycles in our model, it cannot be considered a member of any of the groups (i)-(vi).

\section{Further Questions}

Since the article so far has focused on the need for a knife-edge condition for the technological opportunity-based model to replicate the results of a standard semi-endogenous growth model, and on the oscillatory transition dynamics, there are some questions which we left unanswered. An interesting issue is the contribution of technological opportunity to growth. If this contribution proves to be large, then further empirical investigations should try to address this issue. Another of the essential unanswered questions is the problem of finding a tractable decentralization of the technological opportunity-based model which would enable welfare analysis. Yet another related issue is concerned with capturing technological opportunity empirically. The final question deals with the social planner outcome when the external returns to radical and incremental innovations are not equal, $\mu \neq \nu$. Since most of these aspects are beyond the scope of this article, we shall only hint at how they might be approached in future research.

\subsection{The Contribution of Technological Opportunity to Growth}

The contribution of technological opportunity to growth can be assessed as follows. At the highest level, there is of course no direct impact. Indeed, we obtain the following growth rate decomposition (on the BGP): $\hat{y}=\sigma \hat{A}+\alpha \hat{k}$. 
With our calibrated parameters, it follows that the technology share accounts for $\sigma \hat{A} / \hat{y}=(1-\alpha)=64 \%$ of total growth; the capital accumulation share is $\alpha \hat{k} / \hat{y}=\alpha=36 \%$. Having accounted for the total accumulation of physical capital, resulting from an increase in GDP, and taken the BGP identity $\hat{y}=\hat{k}$, we can readily attribute all GDP growth to technology growth. Going one step deeper, though, one realizes that the increments in technology can also be attributed to various variables. We have $\hat{A}=\mu \hat{B}+\beta \hat{L}$. The central issue is that incremental innovations, increasing the stock of knowledge, cannot go on without technological opportunity. However, the share of technological opportunity growth in technology growth is $\mu=50 \%$. The remaining $50 \%$ is attributed to pure population growth, the ultimate source of growth in our model. We can thus conclude that if technology growth is decomposed into its constituent parts, $\sigma \mu \hat{B} / \hat{y}=\mu(1-\alpha)=32 \%$ of total GDP growth can be attributed to the growth in technological opportunity, $\sigma \beta n / \hat{y}=(1-\mu)(1-$ $\alpha)=32 \%$ to pure population growth, and the remaining $\alpha=36 \%$ to capital accumulation. Summing up, given that our baseline parameter choices are accepted, our growth accounting exercise implies that $32 \%$ of GDP growth should be attributed to growth in technological opportunity, which is a large number.

\subsection{Decentralizing the Allocation}

The decentralization of our model ought to handle the value of radical innovations with care. Their value should approximated by the technological opportunity they open up, but the problem is that in direct terms, they have no value. In the decentralized economy, who would then reap the profits from incremental innovations following a given radical innovation? Are patents able to span the whole addition to technological opportunity? How could the profits from technology improvements be shared between radical and incremental innovators in an interior equilibrium? Should the radical innovation sector be, at least partially, funded by the state to obtain a result comparable to the social optimum? We leave these questions open with the hope that they will be addressed in the subsequent research. 


\subsection{An Empirical Approach to Technological Oppor- tunity}

Another difficult issue is how to capture technological opportunity empirically. It is clear that if one accepts technological opportunity as the driving force behind technological advances, then to understand the evolution of economic variables it is not sufficient to look at the evolution of actual technology. One has to look at the evolution of technological opportunity as well. But how would one be able to capture technological opportunity, which is a forward-looking variable? ${ }^{5}$

A hint for a promising approach can be found in the works of Hall, Jaffe and Trajtenberg [12] as well as Trajtenberg, Henderson and Jaffe [34]. Hall, Jaffe and Trajtenberg [12] construct a comprehensive U.S. patent citation dataset. Two indexes, called originality and generality, are of particular interest here. Generality measures how often a patent is cited by subsequent patents that belong to different fields, whereas originality estimates how large the range of fields is that a given patent cites. The idea would then be then that patent citations and the measures of generality and originality can help find a method of discriminating between patents and estimating their impact on technological opportunity.

Patent citations come in two forms, citations made and received. Citations made give information on the amount of prior knowledge a given patent is based on. Citations received give information on how useful the innovation was for other patents. Thus, if a patent receives a citation, then this means the innovation underlying that patent had opened up technological opportunity. The more citations a patent receives, the larger is the amount of technological opportunity opened up. What Hall, Jaffe and Trajtenberg [12] show is that around $80 \%$ of all citations are made during the first 20 years after a patent was accepted. Thus, it seems evident that when an innovation opens up technological opportunity, this opportunity is always finite. This finding seems to provide some support for our approach.

The open question is still how to estimate the degree to which an innovation is radical, and to which it is incremental. We conjecture that the information on patent citations in combination with the indexes of original-

\footnotetext{
${ }^{5}$ Thompson [33] attempts to estimate the elasticities of R\&D production functions and suggests to think about these as measures of technological opportunity. Obviously, this interpretation is not applicable in our case and thus the results obtained are not extendable to the framework presented here.
} 
ity and generality might possess enough information to provide an empirical justification to the proposed division of innovations. We feel that the dataset on U.S. patent citations ought to be utilized for this purpose.

Quantifying technological opportunity is an important question for future research: this concept may be used to discriminate between competing growth theories. In particular, the technological complexity-based literature (e.g. Greenwood and Yorukoglu [11]; Pintea and Thompson [26]) which considers the recent slowdown in U.S. GDP growth to be a transitional effect due to the extensive application of information technologies which are inherently complex, could be given a new basis for empirical comparisons against the semi-endogenous growth literature that links this slowdown to the slowdown in population growth (e.g. Jones [16]) and assumes returns to scale in R\&D to be not constant but decreasing.

\subsection{What if $\mu \neq \nu$ ?}

Here we shall discuss what happens if the magnitudes of external returns to incremental and radical innovations are not equal. The knife-edge condition $\mu=\nu$ forces technology and technological opportunity to grow at equal rates along the BGP which somewhat blurs the clear-cut distinction between these two variables and suggests that if for some reason the condition $\mu=\nu$ was automatic, it would be sufficient to use the reduced-form technology accumulation framework along the lines of Jones [15] for the assessment of long-run economic growth (but not for convergence to the BGP).

Let us now investigate whether a social planner can allocate factors of production across sectors and time in a way that guarantees long-run growth even if $\mu \neq \nu$. The answer to this question is a clear yes, and one possible tool is continuous labor re-allocations. Researchers should be continuously re-allocated from the sector that has higher returns to the sector that has lower returns, making up for the arising deficiency in output of a particular type of innovations.

To help with the intuition, let us take the ratio of incremental to radical innovations, which is given as

$$
\frac{C}{R}=\frac{\delta}{\gamma}\left(\frac{u^{*}}{1-u^{*}}\right)^{\beta} \frac{B^{\mu}}{A^{\nu}},
$$

which implies a growth rate of $\widehat{(C / R)}=\mu \hat{B}-\nu \hat{A} \neq 0$ if labor re-allocations are assumed out. So, if the spillovers in incremental innovations are larger 
than those in radial innovations $(\mu>\nu)$, technological opportunity will be depleted over time and the economy will experience stagnation afterwards. Intuitively, this means that there will be not enough radical innovations (like new product lines or general purpose technologies) to incrementally improve upon. Hence, a social planner who notices that there are insufficient radical innovations ought to re-allocate more researchers towards the sector creating radical innovations. From equation (22), one can directly deduce that continuous shifts of researchers towards radical innovations can, if fast enough, offset the depletion effect due to incremental innovations.

On the other hand, if $\mu<\nu$, then incremental innovations will arrive at a lower pace than radical innovations, ${ }^{6}$ making technological opportunity expand over time faster than technology. This means that more and more opportunities for technological improvements will emerge, which cannot be utilized. Hence, a planner who allocates more and more researchers to incremental innovations over time, will be able to do better than a planner who sticks to a static labor allocation.

Please note that for $\mu \neq \nu$, but with both parameters sufficiently close to each other, the model does not offer a BGP, but preserves its major characteristics by continuity: growth remains semi-endogenous, both kinds of innovations are produced simultaneously, and the potential for cyclical dynamics (dampened oscillations) is preserved. What is changed is the fact that labor allocations and the ratio of technology to technological opportunity are no longer stationary in the long run.

Of course, we did not evaluate the optimal social planner solution for the general case with $\mu \neq \nu$ but merely hinted at the requirements which are sufficient to place the economy on a long-run (unbalanced) growth path. Whether the social planner would optimally chose this kind of re-allocations is a question that requires further investigation.

\section{Conclusion}

In this article we have derived an R\&D-based semi-endogenous growth model where technological advances depend on the available amount of technological opportunity. We distinguish between two types of innovations, which have

\footnotetext{
${ }^{6}$ Of course, initially incremental innovations might arrive faster, but over time radical innovations will for sure overtake.
} 
different impacts on the evolution of knowledge. Incremental innovations provide direct increases to the stock of knowledge but reduce the technological opportunity which is required for further incremental innovations. Radical innovations serve to renew this opportunity. Hence, technological opportunity behaves like a renewable resource. Even though the basic idea follows Olsson [23], we generalize his framework in order to incorporate Jones' [15] and Romer's [27] models and to compare under what subset of parameter conditions these models predict the same outcomes as ours.

We analyze the model for its growth implications, leading to two novel observations. One, it predicts long-run growth along the lines of Jones [15], Kortum [21], and Segerstrom [29] only if a specific knife-edge condition holds. This condition requires incremental and radical innovations to arrive at the same rates, and thus imply that technology and technological opportunity grow at the same speed. If one expects that this condition is satisfied, then the analytically more tractable model of e.g. Jones [15] might be sufficient to estimate the growth effects of technological progress. If, however, one presumes that it is unlikely that this condition holds, then, of course, our model can help derive the long-run predictions. For example, as we have shown, economic growth can easily come to a halt if technological opportunity is not renewed sufficiently fast because e.g. the technology spillovers in the radical innovations sector are too small.

The second novel result is with respect to the transition. We focus on the particular knife-edge conditions in order to demonstrate that, even though the long-run implications of this model will then be the same as those in Jones [15] or Segerstrom [29], the transition need not. We work out the transition from the social planner solution of our model and show the conditions when it need not be monotonic; on the contrary, we obtain endogenous oscillations and limit cycles for a wide range of plausible parameter values. We therefore suggest that technological opportunity, as characterized here, can be a source of endogenous cycles without needing uncertainty.

Finally, we present the possible directions for future research. These include decentralizing the technological opportunity-based setup and quantifying technological opportunity empirically. Clearly, these extensions give rise to several complications which ought to be discussed in detail.

As a final note we would like to advocate the idea of technological opportunity as a concept which is extremely useful for the description of the evolution of technology. This is a rather novel idea which still lacks a sound empirical justification, but we are convinced that it will gradually earn its 
deserved place in the theory of economic growth and technical change.

\section{References}

[1] P. Aghion and P. Howitt. A Model of Growth Through Creative Destruction. Econometrica, 60(2):323-351, 1992.

[2] B. Amable. Croissance et cycles induits par les innovations radicales et incrémentales. Annales d'Economie et Statistique, 44:91-109, 1996.

[3] L. G. Arnold. The Dynamics of the Jones' R\&D Model. Review of Economic Dynamics, 9:143-152, 2006.

[4] T. F. Bresnahan and M. Trajtenberg. General Purpose Technologies: Engines of Growth? Journal of Econometrics, 65:83-108, 1995.

[5] F. Caselli and W. J. Coleman. The World Technology Frontier. American Economic Review, page forthcoming, 2006.

[6] L. K. Cheng and E. Dinopoulos. Schumpeterian Growth and Stochastic Economic Fluctuations. mimeo, University of Florida, 1992.

[7] T. S. Eicher and S. J. Turnovsky. Convergence in a Two-Sector Nonscale Growth Model. Journal of Economic Growth, 4(4):413-428, 1999.

[8] P. Francois and H. Lloyd-Ellis. Animal Spirits Through Creative Destruction. American Economic Review, 93(3):530-550, 2003.

[9] S. Freeman, D. Hong, and D. Peled. Endogenous Cycles and Growth with Indivisible Technological Developments. Review of Economic Dynamics, 2(2):402-432, April 1999.

[10] R. M. Goodwin. A Growth Cycle, [in:] C.H. Feinstein, ed.: Socialism, Capitalism, and Economic Growth, 1967.

[11] J. Greenwood and M. Yorukoglu. 1974. Carnegie-Rochester Series on Public Policy, 46:49-95, 1997.

[12] B. H. Hall, A. B. Jaffe, and M. Trajtenberg. The NBER Patent Citation Data File: Lessons, Insights and Methodological Tools, 2001. 
[13] E. Helpman and M. Trajtenberg. A Time to Sow and a Time to Reap: Growth Based on General Purpose Technologies. NBER Working Paper, 4854, 1994.

[14] P. Howitt. Steady Endogenous Growth with Population and R\&D Inputs Growing. Journal of Political Economy, 107(4):715-730, 1999.

[15] C. I. Jones. R\&D-Based Models of Economic Growth. Journal of Political Economy, 103(4):759-84, 1995.

[16] C. I. Jones. Sources of U.S. Economic Growth in a World of Ideas. American Economic Review, 92(1):220-239, 2002.

[17] C. I. Jones. Growth and ideas. In P. Aghion and S. Durlauf, editors, Handbook of Economic Growth. North-Holland, 2005.

[18] C. I. Jones. The Shape of Production Functions and the Direction of Technical Change. Quarterly Journal of Economics, 120(2):517-549, 2005.

[19] B. Jovanovic and R. Rob. Long Waves and Short Waves: Growth through Intensive and Extensive Search. Econometrica, 58(6):13911409, 1990.

[20] B. Jovanovic and P. L. Rousseau. Two Technological Revolutions. Journal of the European Economic Association, 1(2-3):419-428, 2003.

[21] S. S. Kortum. Research, Patenting, and Technological Change. Econometrica, 65(6):1389-1419, 1997.

[22] F. E. Kydland and E. C. Prescott. Time to Build and Aggregate Fluctuations. Econometrica, 50(6):1345-1370, 1982.

[23] O. Olsson. Technological Opportunity and Growth. Journal of Economic Growth, 10(1):31-53, 2005.

[24] P. F. Peretto. Technological Change and Population Growth. Journal of Economic Growth, 3(4):283-311, 1998.

[25] K. L. Phillips and J. Wrase. Is Schumpeterian "Creative Destruction" a Plausible Source of Endogenous Real Business Cycle Shocks? Journal of Economic Dynamics and Control, 30:1885-1913, 2006. 
[26] M. I. Pintea and P. Thompson. Technological Complexity and Economic Growth. Review of Economic Dynamics, 10:276-293, 2007.

[27] P. M. Romer. Endogenous technological change. Journal of Political Economy, 98(5):S71-102, 1990.

[28] P. M. Romer. Two Strategies for Economic Development: Using Ideas and Producing Ideas. Proceedings of the World Bank Annual Conference on Development Economics, 1992, pages 63-115, 1993.

[29] P. S. Segerstrom. Endogenous Growth without Scale Effects. The American Economic Review, 88(5):1290-1310, 1998.

[30] P. S. Segerstrom. The Long-Run Growth Effects of R\&D Subsidies. Journal of Economic Growth, 5(3):277-305, 2000.

[31] T. M. Steger. Non-Scale Models of R\&D-Based Growth: The Market Solution. Topics in Macroeconomics, 5, 2005.

[32] H. Strulik. The Role of Human Capital and Population Growth in $\mathrm{R}$ \& D-based Models of Economic Growth. Review of International Economics, 13(1):129-145, 2005.

[33] P. Thompson. Technological Opportunity and the Growth of Knowledge: A Schumpeterian Approach to Measurement. Journal of Evolutionary Economics, 6:77-97, 1996.

[34] M. Trajtenberg, R. Henderson, and A. B. Jaffe. University Versus Corporate Patents: A Window on the Basicness of Inventions. Center for Economic Policy Research, Stanford University, 1994.

[35] M. L. Weitzman. Recombinant Growth. The Quarterly Journal of Economics, 113(2):331-360, 1998. 
Département des Sciences Économiques de l'Université catholique de Louvain

Institut de Recherches Économiques et Sociales

Place Montesquieu, 3

1348 Louvain-la-Neuve, Belgique 